\title{
GROWTH AND PROPERTIES OF QUASIPERIODIC HETEROSTRUCTURES
}

\author{
Roy CLARKE, J. TODD *, R. MERLIN and K. BAJEMA \\ Department of Physics, The University of Michigan, Ann Arbor, Michigan 48109, USA
}

and

\section{P.K. BHATTACHARYA and F.-Y. JUANG}

Solid State Electronics Laboratory, Department of Electrical Engineering and Computer Science, The University of Michigan, Ann Arbor, Michigan 48109, USA

\begin{abstract}
We have recently demonstrated the MBE growth of heterostructures in which layers of GaAs and AlAs were deposited in a Fibonacci sequence. This yields a quasiperiodic structure with the ratio of incommensurate periods equal to the golden mean, $\tau$. We present an overview of the unique structural, electronic, and vibrational properties of this new class of materials emphasizing the role of the incommensurate structure normal to the layers. Inevitably, defects are introduced by growth fluctuations but do not appear to disrupt significantly the special characteristics which originate from the quasiperiodic ordering.
\end{abstract}

\section{Introduction}

The MBE technique lends itself ideally to the artificial structuring of layered materials on nanometer length scales. Over the past fifteen years much effort has gone into making heterostructures which have periodically repeating layers, i.e., superlattices. Recently, a new class of heterostructure was demonstrated in which the constituent layers are deposited not in a periodic fashion but according to a predetermined, yet non-repeating, mathematical sequence based on the Fibonacci series [1]. This yields a so-called "quasiperiodic" (or incommensurate) structure with very interesting and unusual properties.

It has long been recognized that quasiperiodic ordering could offer interesting possibilities for experimental studies of novel physical phenomena [2]. The motivation for this was, in large part, theoretical work on quasiperiodic one-dimensional (1D) wave equations revealing spectra and eigenstates state are quite unlike those of periodic or

\footnotetext{
* Present address: Cavendish Laboratory, University of Cambridge, Cambridge, CB3 0HE, UK.
}

random 1D systems [3]. The major problem in fabricating quasiperiodic structures has been the fact that simple incommensurate modulations require increasingly larger layer thicknesses to approach the irrational limit. Layer deposition in sequences generated by special growth rules provides a solution to this problem [1]. Heterostructures grown according to these sequences show a degree of quasiperiodicity that is determined not by the width of individual layers (which is arbitrary), but by the thickness of the sample [1].

A number of studies [5-9] have focussed on the class of structures derived from the Fibonacci sequence. The general properties of these materials and results of our specific experiments on Fibonacci $\mathrm{GaAs}-\mathrm{Al}_{x} \mathrm{Ga}_{1-x}$ As heterostructures are discussed in this paper.

\section{Structural properties and X-ray scattering}

One way to make a quasiperiodic heterostructure is to deposit the layers in a Fibonacci series such that each succeeding generation of the series is the sum of the previous two. Accordingly, the 
arrangement of layers is built up as follows:

A

$\mathrm{AB}$

$\mathrm{ABA}$

$\overline{\mathrm{AB}} \overline{\mathrm{A}} \mathrm{AB}$

$\overline{\mathrm{ABA}} \overline{\mathrm{AB}} \mathrm{ABA}$

$\overline{\mathrm{ABAAB}} \overline{\mathrm{ABA}} \mathrm{ABAAB}$

...etc.

In order to obtain a Fibonacci superlattice, one simply replaces A and B by two arbitrary blocks of layers $[1,7]$. The resulting structure is shown schematically in fig. 1 . This arrangement leads to a structure which shows two basic reciprocal periods in a ratio given by the golden mean $\tau=(1$ $+\sqrt{5}) / 2$, perpendicular to the layers [1]. The associated structure factor consists of a dense set of components such that diffraction peaks are expected at wavevectors given by $k=2 \pi d^{-1}$ ( $m+$ $n \tau)$, where $m$ and $n$ are integers and $d=\tau d_{\mathrm{A}}+d_{\mathrm{B}}$;

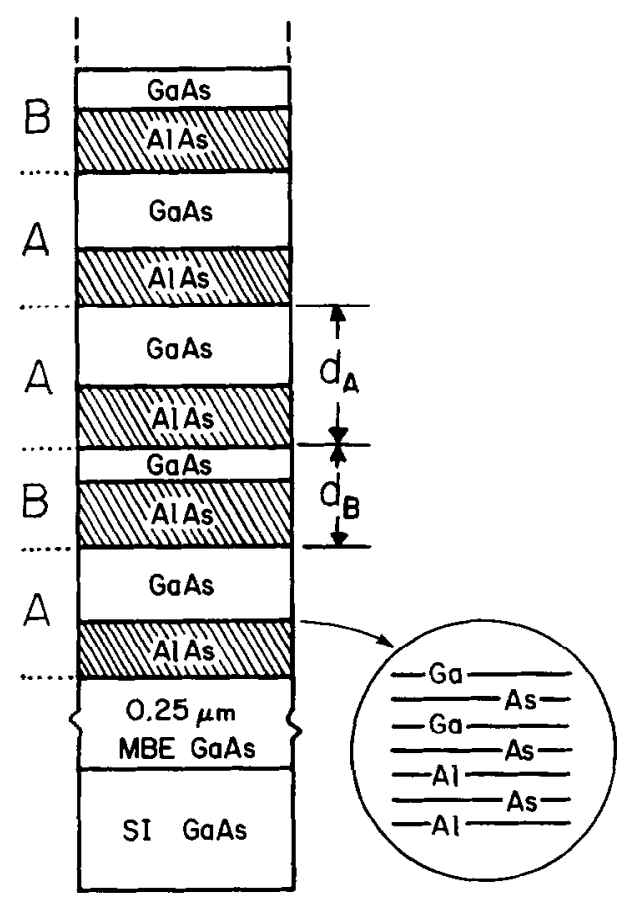

Fig. 1. Schematic arrangement of GaAs and AlAs layers in a Fibonacci sequence ABAABABA... For the sample considered in section 2, the AlAs strata are nominally of identical thicknesses $(\sim 17 \AA) ; d_{\mathrm{A}}=59 \AA, d_{\mathrm{B}}=37 \AA$.
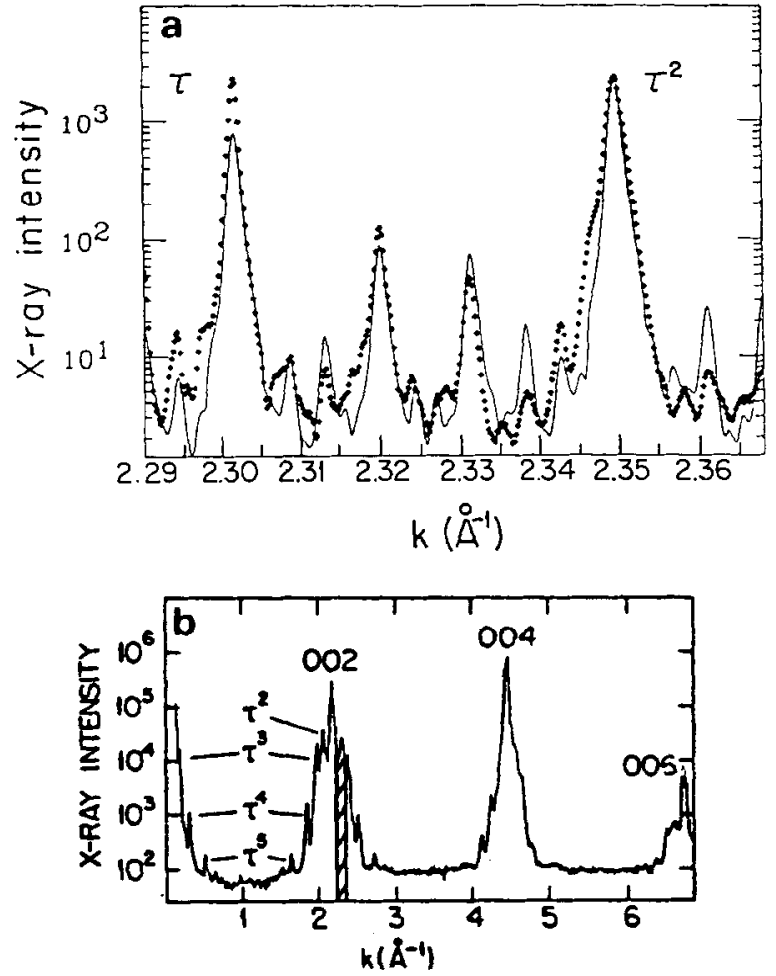

Fig. 2. (a) Synchrotron X-ray data (dots) and calculated diffraction profile for the ideal Fibonacci structure (solid line); the sample is described in section 2. (b) Indexing of strong peaks in a low-resolution scan. Shaded region shown expanded in (a).

$d_{\mathrm{A}}$ and $d_{\mathrm{B}}$ are the thicknesses of the building blocks $\mathrm{A}$ and $\mathrm{B}[1]$.

$X$-ray diffraction patterns have been obtained [4] from a Fibonacci heterostructure consisting of $\mathrm{A} \equiv[17 \AA \mathrm{AlAs}-42 \AA \mathrm{GaAs}]$ and $\mathrm{B} \equiv[17 \AA$ AlAs-20 $\AA$ GaAs]; the sample was grown by molecular beam epitaxy on a (001) GaAs substrate. The patterns for $\boldsymbol{k}$ parallel to [001], in fig. 2 , demonstrate many of the unusual properties of quasiperiodic ordering. The synchrotron X-ray data indicate that, at least up to the instrumental resolution $(\simeq 0.0015 \AA \mathrm{FWHM})$, the diffraction peaks do indeed form a dense set. Moreover, the measurements agree remarkably well with the calculated profile for an ideal Fibonacci structure (solid curve in fig. 2) [4]. This, and also numerical simulations [4], show that quasiperiodic ordering is largely insensitive to the unavoidable random fluctuations in the growth parameters such as the 
deposition fluxes and the timing of the source shutters. A further interesting effect is observed in the extended low-resolution scan of fig. $2 b$; the dominant zinc-blende peaks and their satellites can be expressed as $k=2 \pi d^{-1} \tau^{p}$, with integer $p$. This behavior results from $d_{\mathrm{A}} / d_{\mathrm{B}}$ being close to $\tau$ in our sample [4]. It is not a general feature of Fibonacci heterostructures.

\section{Electronic and optical properties}

Tight-binding models describing electrons in Fibonacci structures have been studied by several authors [6-9]. The spectrum is a Cantor set characterized by clusters of eigenvalues that divide into three subclusters [6-9]. The wavefunctions are critical (i.e., neither localized nor extended) exhibiting either self-similar or chaotic behavior $[8,9]$. This applies only to bulk states; finite samples can further show solutions localized at the surfaces [8].

Investigations of the electronic structure of Fibonacci $\mathrm{GaAs}-\mathrm{Al}_{x} \mathrm{Ga}_{1-x}$ As heterostructures using standard optical probes reveal mainly excitonic features. The example of a structure with $\mathrm{A} \equiv\left[20 \AA \mathrm{Al}_{0.3} \mathrm{Ga}_{0.7} \mathrm{As}-40 \AA \mathrm{GaAs}\right]$ and $\mathrm{B} \equiv[20$ $\left.\AA \mathrm{Al}_{0.3} \mathrm{Ga}_{0.7} \mathrm{As}-20 \AA \mathrm{GaAs}\right]$ is given in fig. $3 \mathrm{a}$. Fig. $3 b$ shows results of effective-mass calculations of the electron, heavy-hole and light-hole spectra of this sample, for motion normal to the layers. These results were used to further determine the $\operatorname{Im}(\chi)$ versus photon-energy plots shown in fig. 3a. The comparison with the experimental data indicates a correlation between the positions of the exciton peaks and the largest calculated plateaus; the latter reflect major gaps in the $1 \mathrm{D}$ spectrum of fig. $3 \mathrm{~b}$. Since surface states may also occur at these gaps, it is not clear whether the excitons derive from bulk critical states or from quasi-2D states localized at the surface.

\section{Raman scattering by acoustic phonons and vibra- tional properties}

The spectrum of phonons in 1D Fibonacci lattices shows a self-similar hierarchy of gaps which decrease in size with the phonon frequency $[6,8,9]$. As expected, the eigenfunctions are extended in the continuum limit. Their high-frequency behavior is not as yet well understood although there is some evidence favoring localization [6]. If this turns out to be correct, a transition between extended and localized states may take place at intermediate frequencies.

Raman scattering has been used to study longitudinal acoustic (LA) phonons propagating parallel to the growth axis in Fibonacci $\mathrm{GaAs}-\mathrm{Al}_{x} \mathrm{Ga}_{1-x} \mathrm{As}$ heterostructures [5]. The re-
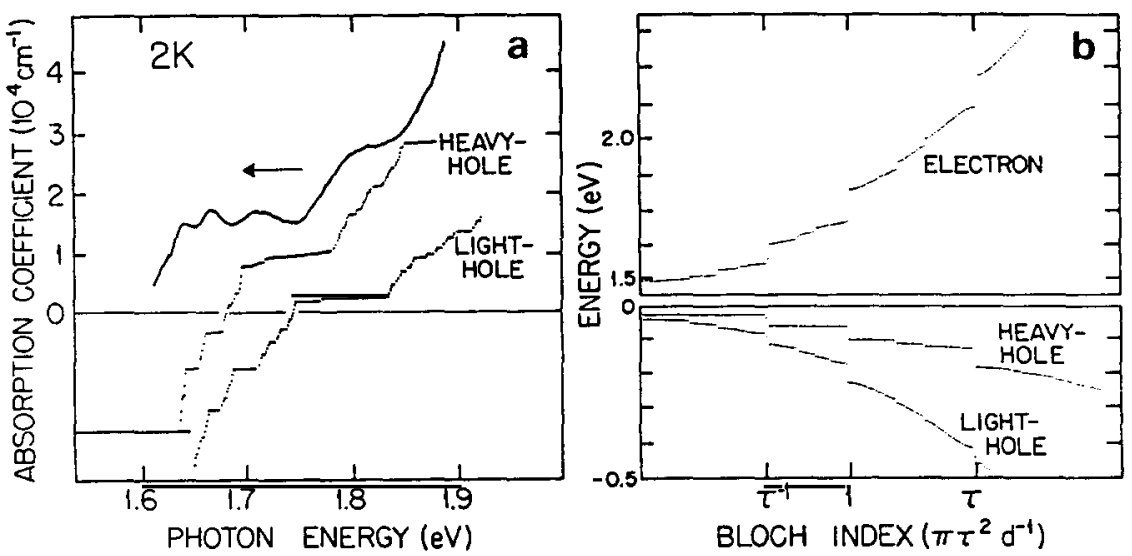

Fig. 3. (a) Optical absorption coefficient and calculated $\operatorname{Im}(\chi)$ for transitions involving heavy- and light-hole states, in arbitrary units. (b) Energy versus Bloch index. Heterostructure parameters are indicated in section 3. 


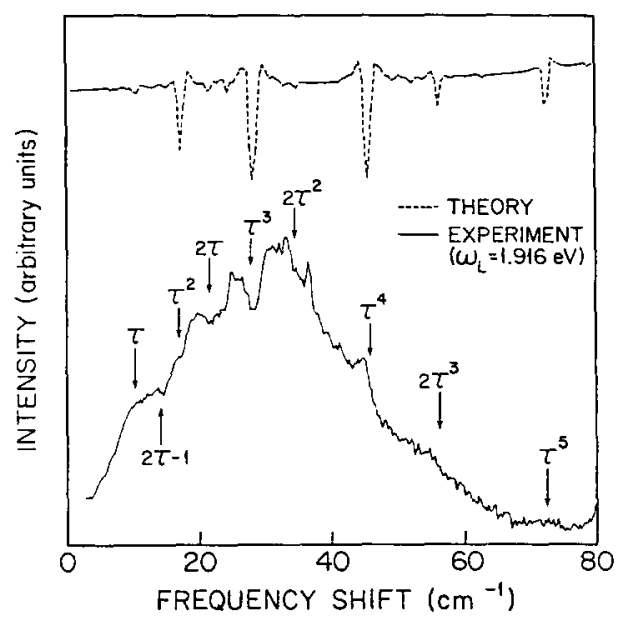

Fig. 4. Room temperature resonant Raman spectrum of the heterostructure considered in section 4 corrected for thermal factors, and calculated density of states of LA modes propagating along [001] (dashed curve). Arrows denote expected midfrequencies of main gaps in units of $\pi c d^{-1} ; c$ is the average sound velocity.

sults reveal important differences between resonant and non-resonant spectra. For the latter, the scattering is largely determined by structural effects as in the case of periodic superlattices [10]. Off-resonance data from the sample described in section 2 show doublets centered at frequencies that follow a $\tau^{p}$ behavior [5], consistent with the $X$-ray findings. The Raman spectrum of the same sample obtained under resonant conditions is shown in fig. 4. The scattering reflects now a weighted density of states of LA modes, providing an experimental demonstration of the richness of the phonon spectrum. Resonances with electronic states localized at the surface can possibly account for this behavior [5].

\section{Acknowledgements}

The contributions of K.M. Mohanty and J.D. Axe to the synchrotron results are gratefully acknowledged. The research was supported in part by ARO Contracts DAAG-29-83-K-0131 and DAAG-29-85-K-0175.

\section{References}

[1] R. Merlin, K. Bajema, R. Clarke, F.Y. Juang and P.K. Bhattacharya, Phys. Rev. Letters 55 (1985) 1768.

[2] J.B. Sokoloff, Phys. Rev. B22 (1980) 5823; S. Das Sarma, A. Kobayashi and R.E. Prange, Phys. Rev. Letters 56 (1986) 1280.

[3] See, e.g., S. Ostlund and R. Pandit, Phys. Rev. B29 (1984) 1394.

[4] J. Todd, R. Merlin, R. Clarke, K.M. Mohanty and J.D. Axe, to be published.

[5] K. Bajama and R. Merlin, to be published.

[6] J.P. Lu, T. Odagaki and J.L. Birman, Phys. Rev. B33 (1986) 4809.

[7] T. Odagaki and L. Friedman, Solid State Commun. 57 (1986) 915.

[8] F. Nori and J.P. Rodriguez, to be published.

[9] M. Kohmoto and J.R. Banavar, to be published; see also M. Kohmoto, Phys. Rev. Letters 51 (1983) 1198.

[10] See, e.g., C. Colvard, T.A. Gant, M.V. Klein, R. Merlin, R. Fischer, H. Morkoc and A.C. Gossard, Phys. Rev. B31 (1985) 2080. 